\title{
BMJ Open Influencing factors on compliance of timely visits among patients with proliferative diabetic retinopathy in southern China: a qualitative study
}

Fang Duan, ${ }^{1}$ Yuhong Liu, ${ }^{1}$ Xiang Chen, ${ }^{1}$ Nathan Congdon, ${ }^{1}$ Jian Zhang, ${ }^{1}$
Qianyun Chen, ${ }^{1}$ Lingling Chen, ${ }^{1}$ Xi Chen, ${ }^{2}$ Xiulan Zhang, ${ }^{1}$ Chengpu Yu, ${ }^{2}$ Yizhi Liu ${ }^{1}$

To cite: Duan F, Liu Y, Chen $\mathrm{X}$, et al. Influencing factors on compliance of timely visits among patients with proliferative diabetic retinopathy in southern China: a qualitative study. BMJ Open 2017:7:e013578.

doi:10.1136/bmjopen-2016013578

- Prepublication history for this paper is available online. To view these files please visit the journal online (http://dx.doi.org/10.1136/ bmjopen-2016-013578).

$\mathrm{YL}$ and $\mathrm{CY}$ contributed equally to this work.

Received 28 July 2016 Revised 28 January 2017 Accepted 6 March 2017

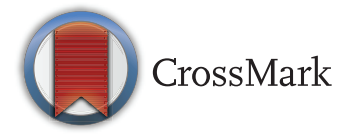

${ }^{1}$ Zhongshan Ophthalmic Centre, State Key Laboratory of Ophthalmology, SunYatsen University, Guangzhou, China

${ }^{2}$ Centre of Medical Anthropology and Behavioural Health, School of Sociology and Anthropology, Sun Yat-sen University, Guangzhou, China

Correspondence to Dr Yizhi Liu; yzliu62@yahoo.com

\section{ABSTRACT}

Objective: To identify the reasons for low adherence among patients with diabetic retinopathy (DR) in southern China using a qualitative method.

Methods: Exploratory indepth interviews were conducted in 27 diabetic patients with proliferative diabetic retinopathy who required vitrectomy surgery at Zhongshan Ophthalmic Centre, Sun Yat-sen University, from March to August 2015. Qualitative data analysis and research software (ATLAS.ti7) was used for data processing and analysis.

Results: Factors influencing the occurrence of timely visits included lack of DR related knowledge, fear and worries about insulin, interactions between patients and society combined with the complexity of emotions and social culture, and the economic burden of treatment.

Conclusions: Although the reasons for low adherence involved social, emotional, cultural and economic factors, the key issue was the lack of awareness and knowledge of DR. Our findings have several practical implications for health policymakers and programme planners in China.

\section{INTRODUCTION}

The prevalence of diabetes mellitus (DM) in China has increased nearly 10 -fold to $11.6 \%$ from 1980 to $2010 .^{1}$ China has the largest number of people with DM in the world (113.9 million). However, less than a third have been diagnosed. Furthermore, $50.1 \%$ of the Chinese adult population may have prediabetes, ${ }^{1}$ an important risk factor for the development of overt DM. With the aging of the Chinese population, the number of patients with DM is expected to double from 2000 to $2030 .^{2}$ Therefore, DM and its complications is presenting a major public health problem in China.

Diabetic retinopathy (DR) is a leading cause of blindness worldwide, ${ }^{3}{ }^{4}$ and a priority

\section{Strengths and limitations of this study}

- This is the first study to use qualitative method to study adherence of diabetic retinopathy patients in China.

- Indepth interview of the patients and their family members simultaneously allowed us to understand the interaction between them.

- The study was conducted by anthropologists and ophthalmologists working together, which allowed us to investigate this complex issue from multiple angles.

- Our study was limited by using a hospital based selected sample. All selected participants developed proliferative diabetic retinopathy prior to the interview.

disease in the 'VISION 2020' initiative for the global elimination of preventable blindness. Population based research in the USA showed that the incidence of DR among patients with DM aged $>40$ years was $28.5 \%$. Vision threatening DR accounts for $4.4 \%$ of cases and, of those, proliferative diabetic retinopathy (PDR) accounts for $1.5 \% .^{5}$ Nearly half of people with diabetes $(43.1 \%)$ in a population based rural Chinese study in Handan had DR, while $6.3 \%$ had vision threatening DR. ${ }^{6}$

Two population based studies in Beijing identified the population prevalence of DR as $6.5 \%$ in $2001^{7}$ and $24.7 \%$ in $2009 .^{8}$ These two studies showed that the prevalence of DR in China is increasing. As the average lifespan of the Chinese population extends and the prevalence of DM increases, the prevalence of DR will also increase. Therefore, the urgent problem is how can we implement early DR interventions and reduce the corresponding loss of labour and incurred medical expenditures.

It has been suggested in Chinese guidelines for the prevention and treatment of DR 
that patients with DM should receive a dilated fundus examination once a year. ${ }^{9} 10$ However, two-thirds of patients with DM in China have not undergone an examination for more than 1 year, and nearly half $(43.2 \%)$ have never been examined. ${ }^{11}$ Many adults living with DM remain unaware of their ocular condition until their DR has progressed to a stage at which treatment is difficult. ${ }^{12}$ This phenomenon is more severe in rural areas. Only $54.5 \%$ of patients with DR who need panretinal photocoagulation have completed treatment. ${ }^{13}$ Currently, there is no comprehensive national diabetes prevention or screening system available in China, although the government is hoping to establish a chronic disease prevention service programme gradually; it will take some time to see its effects. It is important to identify the reasons for this very low adherence with the recommended treatment, to seek solutions to improve the situation and to reduce the burden of blindness due to DR.

In previous questionnaire based studies of adherence with DR care in China, several factors were identified, such as lack of knowledge about the condition, older age, low income, inadequate education, lack of health insurance and transportation. ${ }^{11} 1415$ However, quantitative studies have their limitations. Qualitative research is complementary to quantitative research methods and is used in small groups of patients to explore complex issues in greater depth, allowing findings and themes to emerge from the data rather than testing predetermined hypotheses. ${ }^{16}$

In our study, we conducted indepth interviews with patients with PDR who did not adhere to timely treatment and developed the need for vitrectomy. Our aim was to explore possible intervention methods to prevent the occurrence of DR, and to lay the foundations for future intervention studies.

\section{RESEARCH DESIGN AND METHODS}

Topic guides were preliminarily formed based on previous literature ${ }^{11}{ }^{17-19}$ and discussion with experienced ophthalmologists and anthropology experts; the final versions were developed after the first five patients. All indepth interviews were conducted with diabetic patients with PDR who required vitrectomy surgery at Zhongshan Ophthalmic Centre, Sun Yat-sen University. Inclusion criteria included PDR patients who were older than 18 years and had not received a dilated fundus examination for over 1 year before developing PDR.

Ethics approval was obtained from the ethics committee of Zhongshan Ophthalmic Centre (2015MEKY067). After being informed about the objectives of the study, informed consent was obtained from all participants who were assured of confidentiality, de-identification of all data and adequate protection against release of confidential information. Recorded interviews of approximately half an hour in length were conducted by a professor (CY) of anthropology and an undergraduate student (XC) from the anthropology department, from May to August 2015.

The interview methods have been described elsewhere. ${ }^{20}$ In brief, consecutive patients were interviewed with or without accompanying family members, according to patients' willingness. The audio recorded interview data were transcribed verbatim to capture the cultural concepts and nuances embedded in the language. The transcripts were reviewed to ensure accuracy (by LC and XC, with qualitative and ethnographic training). In the process of analysis, the code lists were compared to resolve any discrepancies (by FD and XC). Once agreement had been achieved, a coding framework was developed to capture key themes, and each coded theme was subjected to further analysis to identify subthemes and illustrative quotes. All of the above steps were performed using software for qualitative data processing and analysis (ATLAS.ti7). To protect family members' anonymity, unique identifiers were used.

\section{RESULTS}

We screened 30 patients with PDR, and 27 agreed to participate in the interviews, including 26 patients with type $2 \mathrm{DM}$ and 1 patient with type $1 \mathrm{DM}$. Of these, 20 patients were accompanied by family members during the interviews. The demographic characteristics of the 27 patients are shown in table 1 . The frequency of the key words that emerged from the interviews are listed in table 2.

\section{Knowledge of DR and need for information on DR}

From quantitative studies it has been found that patients with DR generally lack DR related knowledge, ${ }^{11}{ }^{21}$ which is consistent with what we found in our interviews. Patient No 16 was a 26-year-old unmarried nurse at a township hospital and visited the doctor with her 60-year-old father. There was no history of DM in her family. She was found to have type $1 \mathrm{DM}$ when she was 7 years old. When asked about how she viewed her DM complications, she said:

Table 1 Demographic characteristic of the 27 diabetes mellitus patients with proliferative diabetic retinopathy

\begin{tabular}{ll}
\hline Characteristic & Value \\
\hline Male sex (n (\%)) & $13(48.1)$ \\
Age (years) (mean \pm SD, range) & $53.1 \pm 11.1,32-71$ \\
Married (n (\%)) & $25(92.6)$ \\
Duration of DM (years) (mean $\pm S D$, & $10.9 \pm 7.6,0.2-28$ \\
range) & \\
Occupation (n (\%)) & \\
$\quad$ Professional (health, education, etc) & $2(7.4)$ \\
$\quad$ Semiskilled (office worker) & $5(18.5)$ \\
$\quad$ Unskilled & $3(11.1)$ \\
$\quad$ Shopkeeper/business & $5(18.5)$ \\
$\quad$ Agriculture & $5(18.5)$ \\
$\quad$ Retired & $4(14.8)$ \\
$\quad$ Unemployed & $3(11.1)$ \\
\hline DM, diabetes mellitus. &
\end{tabular}


Table 2 Frequency of the key words that emerged from the interviews

\begin{tabular}{lc}
\hline Theme & Frequency \\
\hline Lack of DR knowledge & 26 \\
Fear, worry and misunderstanding about the & 6 \\
use of insulin & \\
Interactions between patients and society & 18 \\
Family & 4 \\
Fellow patients & 3 \\
Working & 24 \\
Economic burden of DM and DR treatment & \\
\hline DM, diabetes mellitus; DR diabetic retinopathy.
\end{tabular}

"I did not know DR existed, and no one reminded me to undergo fundus examinations. I have looked up some knowledge on the complications of DM. However, I never thought that the complications would occur to my eyes; in my opinion, they generally occur to the heart and kidney, which affects one's life and must be attended to first. People may pay less attention to the eyes."

This patient was a medical worker, had some DM related knowledge and the possibility of some occurrence of complications, but knew nothing about eye complications. This suggests that DR is not familiar to patients and is not taken as seriously as other life threatening complications. Additionally, this patient, as a medical worker, had no knowledge of DR, which indicates that local medical workers might also lack sufficient knowledge on DR. We also found in our interviews that most patients knew they should regularly have their blood sugar monitored but did not know the potential eye complications and the importance of regular fundus examinations. Of the 27 patients in our study, only one patient (patient No 9) explicitly mentioned that her doctor told her about DR and suggested that she should receive an eye examination annually when she was diagnosed with DM. This suggests the need for patients with DM to improve their DR related knowledge and for the awareness and importance of DR to be strengthened.

Because the patients lacked knowledge of DR and were unaware of the importance of periodic fundus examinations, they did not see their doctor when ocular symptoms occurred. They thought they may have been due to other common non-serious eye diseases, such as myopia or cataracts. For example, patient No 26, a university teacher in Guangdong, often needed to search for documents on the computer. He did not pay attention when his vision became blurry he as thought it was because he was too tired. The eyesight in one of his eyes worsened dramatically from 1.0 to 0.1 within 2 months. A 60-year-old patient (patient No 10) used a few eyedrops when his vision became cloudy. Later, his family thought it was a cataract and sent him to the hospital until his right eye deteriorated due to secondary glaucoma and he experienced headaches. A 32-year-old patient (patient No 11) thought that it was the progression of myopia that caused her blurred vision. Furthermore, three patients (patient Nos 2, 11 and 19) were first diagnosed with DM only after identifying the occurrence of serious PDR affecting their eyesight.

These patients manifested successive symptoms of DR, but their lack of knowledge and awareness of DR resulted in PDR diagnoses when they visited the hospital. This not only leads to treatment difficulties but also affects the prognosis of the disease. Therefore, patients were recommended to seek medical help when noticing blurred vision.

Fear, worry and misunderstanding about the use of insulin Insulin is the most effective hypoglycaemic agent and is widely used in the treatment of patients with DM to reduce their long term complications. We found in our study that some patients had major concerns, or even misunderstood the use of insulin.

Patient No 27, a 60-year-old woman, demonstrated apparent fear when she gave her opinions on using insulin as a neighbour had died because of the frequent use of insulin.

"There may be other reasons for this, but we still resist and dare not to use insulin".

The patient connected the death of a neighbour with the use of insulin, and had a great misunderstanding of the use of insulin, which affected her adherence to insulin use, and led to PDR.

Patient No 26 refused to use insulin because of concerns about insulin dependence and only agreed to use insulin when his oral medicine had become completely ineffective. Now he thinks that his DR was mainly caused by the poor control of his blood sugar caused by the delay in the use of insulin.

"If I had used insulin earlier, my DR may have been better".

Patient No 2, a 71-year-old, farmer, was diagnosed with DM when she went to the hospital because her vision was blurred. She said,

\footnotetext{
"the doctor suggested that I use insulin when my oral medicine could not control my blood sugar. I worry about insulin dependence too, but there is no other way".
}

In addition, the patient worried about the inconvenience of insulin injections because she could not perform the injections on her own due to her poor eyesight.

The main concern regarding the use of insulin was fear of insulin dependence and its side effects. These cases indicate that lack of insulin related knowledge in some patients resulted in insulin use disorders or poor adherence. These concerns are likely to affect patients' use of insulin, resulting in poor control of blood sugar. ${ }^{22}$ 
Intensive therapy with insulin in patients with type 1 diabetes was associated with a substantial reduction in the long term risk of ocular surgery. ${ }^{23}$ Therefore, it is necessary for medical staff to help patients objectively understand this so that patients' unnecessary concerns can be reduced and adherence can be improved, achieving appropriate control of blood sugar, and thereby reducing the occurrence and development of PDR.

\section{Interactions between patients and society involve the complexities of emotion and social culture, presenting a double-edged sword}

As part of social cultures, social relationships can directly affect people's lifestyles and psychological states. Social relationships consist of many types. Family relationships, working relationships and fellow patients' relationships were mentioned most often by patients during the interviews. The influence of these relationships on the development of disease had two sides.

Patient No 7, a 57-year-old man, enjoyed eating and drinking with friends in teahouses. His family began to supervise his life and diet after he was diagnosed with PDR. His wife ( 57 years old) told us

\section{"He used to eat sweets and fats. Now I do not allow him to eat these foods. If a man is not good, the whole family worries about him".}

She always reminded her husband to control his diet. This case is an example of the positive influence of family support in the treatment of disease.

The mother of patient No 16, the only patient with type $1 \mathrm{DM}$, stopped working and took care of patient No 16. The mother reduced the consumption of starchy foods, provided a reasonable mix of meat and vegetables, and increased the quantity of vegetables when taking care of her daughter's diet. The mother looked at many types of folk prescriptions to cure her daughter, including many Chinese herbal medicines which sometimes caused vomiting. The patient said that her mother's actions were useless, even foolish. However, she continued to try what her mother suggested as she understood that her mother was trying to cure her. This case provides an example of the complexity of family relationships and interactions. Such excessive family care may cause a psychological burden.

Patient No 2, a 71-year-old woman, lived in the countryside with her husband. Every time the children returned home, she was busy taking care of them and enjoyed the rare family gatherings. She reluctantly talked about her DM, even when asked by her children. She said,

"I can endure it as long as it is not too severe. I do not want to affect my children's work and life".

With the extension of the average lifespan and low birth rate, China has become an ageing society. Patient
No 2's family represents an example of a lonely elderly family, which is very common. With this type of family structure, the children work outside throughout the year, cannot take good care of their sick parents and often cannot take their parents to hospital in time for examinations and treatment when an illness develops. Simultaneously, the elderly parents are unwilling to trouble their children and become too much of a burden. Therefore, this type of family interaction may delay the treatment of $\mathrm{DM} / \mathrm{DR}$, leading to worsening illnesses.

In addition, some patients often have to engage in social activities because of work, especially if they are men. Some patients are unwilling to tell others about their illness and even

"fear that others know they suffer from the disease" (patient No 25).

Patient No 4, a 52-year-old salesman, often engaged in social activities.

"Once, I was drunk and taken to the hospital. At that time, no one knew that I had DM, and the doctor transfused glucose. I felt more and more uncomfortable and almost lost my life".

After the 'death' experience, patient No 4 no longer concealed his illness, and discovered that his friends understood and considered his lifestyle and diet. This was a great comfort and source of encouragement for him. As a factor, the complexity of social relationships can affect the progress of the disease.

When a person becomes sick, his social relations will expand to his group of fellow patients with the same diseases. Sometimes, the influence of fellow patients is not inferior to that of the family, but it is often overlooked. Patient No 8 initially needed to receive four laser treatment sessions according to his doctor's suggestion, but only one treatment was completed. He decided not to accept the remaining three laser treatment sessions after hearing his fellow patients mention that his eyesight would become worsen. Patient No 21, a businessman, felt thirsty and hungry and lost a substantial amount of weight over 10 years. His friend with DM said that he might have DM and gave him some medicine. Since then, he had been taking the medicine that his friend gave him and had not gone to the hospital for many years. These patients listened to the suggestions of their fellow patients, and missed or delayed their treatment. This suggests a lack of trust between patients and doctors.

Interactions between patients and society involve the influences of emotion and social culture, including all types of social relationships. These influences can be positive or negative. Although people often overlook them, these factors greatly influence the attitudes of patients with DR towards treatment and are sometimes key factor influencing the patient's treatment decision. 
Economic burden of DM and DR treatment

As a chronic disease, people with DM need lifelong medication. In our interviews, most patients had medical insurance but the reimbursement ratios were different by region, and therefore patients reported financial burdens of treating DM, in particular the costs of surgery for treating PDR.

Patient No 10, a 60-year-old woman, was a primary schoolteacher in the town of Guangxi before retirement. She could receive a higher reimbursement ratio if she accepted treatment locally. However, she had to bear all of the treatment costs if she accepted PDR treatment in Guangzhou. Patient No 10's son said,

\begin{abstract}
"a lot of people in our hometown have DM, some have swollen feet and blind eyes, but they are not treated because they cannot afford it. Eventually, they end up not seeing a doctor".
\end{abstract}

Patient No 5, a 51-year-old woman, had DM for 16 years and was living in the countryside. She said that she could not work after she was diagnosed with DM, and her source of income was her husband's small salary.

"For economic reasons, I take cheaper drugs, buy some when we have money, and do not take them when we do not have money".

She also said that farmers in her hometown had rural medical insurance but had to apply for special outpatient reimbursement. Their insurance could reimburse about RMB 100 a month. However, most drug costs and surgery costs could not be reimbursed.

Patient No 14, a 52-year-old driver, was unemployed due to his poor eyesight. In the beginning, when his right eye first became blind, he thought that

"one eye can see, and it doesn't matter if I provide self-care”.

He thought that there were parents and children at home who needed to be taken care of, and he was the main source of income for his family. He did not go to the hospital for treatment until both of his eyes became blind.

"It is a burden for my family. We don't have money and borrowed it from others".

These cases reflect the influence of economic status on patients' behaviour in terms of seeking medical advice. Their illnesses become severe as a result of the delay in treatment for economic reasons. The efficacy and cost effectiveness of early detection and treatment of DR are well established. ${ }^{24}$ It is necessary to emphasise the importance of early detection and treatment.

\section{DISCUSSION}

In our interviews, we found that lack of knowledge of DR was common in patients with PDR and was present in over $90 \%(26 / 27)$ of patients. A previous DR questionnaire study found that a lack of DR knowledge was associated with poor patient adherence, ${ }^{11} 141521$ but it did not explicitly illustrate why patients lacked this knowledge or the missing link that led to the lack of knowledge. We found that the lack of knowledge was due to various reasons. Firstly, DM patients knew that they needed to control their blood glucose but did not know that DM could affect their eyes. This suggests that patients did not obtain information on DR during the course of their diagnosis and treatment of DM. Secondly, patients did not take the initiative to acquire DR knowledge, suggesting that they lacked effective channels for obtaining DM related knowledge. Furthermore, patients who did not know about DR tended to associate their eye problems with other eye diseases that they were more familiar with, such as cataracts or myopia. This suggests that DR remains unfamiliar to the public, although the prevalence of DM is as high as $11.6 \% .{ }^{1}$ In addition, we found that some DM patients had concerns about the use of insulin. Tong et $a l^{22}$ reported that psychosocial and emotional barriers were associated with poor glycaemic control in people with type 2 DM using insulin. Such psychological concerns leading to poor glycaemic control could promote the progression of DR. This also suggests that patients' lack of knowledge is not solely the lack of DR knowledge but may also include lack of knowledge about DM. Campaigns and education that improve awareness of DR may need to be strengthened.

We found that there was a discrepancy between patients' recognition of symptoms and the characteristics of DR. The patients 'see a doctor when they feel ill'. However, the gradual occurrence of DR can easily lead to a gap that results in patients treating DR lightly and ignoring the importance of regular examinations. For patients, the criterion used to judge whether they are healthy is whether their eyesight is affected. There are no obvious symptoms in the early stages of DR. Therefore, patients can easily form the idea that 'DR is not that serious' and thus miss their best treatment opportunity. Previous studies have suggested that patients with less advanced disease and those who do not believe that the disease poses a threat to their vision are less adherent to scheduled follow-up appointments. ${ }^{21} 25$

As an integral component of their life, social relationships affect patients' psychological and behavioural habits. The influence of relationships between patients and society inevitably interacts with the complexity of emotions and social culture and has both positive and negative roles in the patient's disease course. Some families attach great importance to patients after they are diagnosed with PDR, repeatedly reminding them to receive treatment and taking good care of them. Patients may reverse bad habits and actively cooperate 
with treatment due to a sense of responsibility and 'pressure'. However, excessive care can sometimes increase the psychological burden of patients. In other situations, elderly people living alone may delay their treatment because they do not want to affect their children's work and life, and some even conceal the severity of their illness. This demonstrates the emotional and cultural influences in treatment, indicating it is no longer an issue of simply lack of care. There is a contradiction between patients' consideration of their families and the concern for themselves. This also suggests that existing health insurance should provide medical assistance to lonely elderly people when they need it, instead of requiring them to rely on their children. In addition, we found that patients believed their fellow patients more than the doctors. This indicates that fellow patient relationships are an important factor that cannot be ignored. In conclusion, we believe that the treatment and care of patients with DR should be extended to their social relationships to develop more effective treatment measures.

Economic conditions influenced patients' behaviour in receiving medical treatment. More than threequarters of the interviewed patients with PDR reported experiencing economic difficulties and concerns. Some patients concealed their illness, or delayed or did not receive treatment because of economic problems. China is a developing country with a population of 1.3 billion. Per capita gross domestic product is way behind most developed countries, and at present there is no national level medical insurance service available. There is an established socioeconomic gradient in the development of type $2 \mathrm{DM}$ and related complications, with people in lower socioeconomic groups at a higher risk of type 2 DM and its complications. ${ }^{26-28}$ Social and economic problems also manifest in accessibility of medical services. For example, some patients in remote areas chose the nearest hospital for treatment in the early stages but usually encountered limited medical resources and inadequate treatment. This suggests that these patients had difficulty accessing high quality medical care. All of these problems may result in patients with DR failing to be detected at an early stage and failing to be effectively treated.

Early detection of DR is critical, as prompt treatment increases the likelihood of preserving vision. ${ }^{29}$ At present, China lacks an effective system for preventing DR, in contrast with studies in western developed countries. To provide effective care for DR with limited resources, interventions to promote adherence among patients are needed. Adherence is the product of the interaction between various factors. Our study demonstrated the factors influencing the occurrence of timely visits among patients with PDR: lack of DR related knowledge, fear and worries about insulin, the interaction between patients and society combined with the complexity of emotions and social culture, and the economic burden of treatment. Although it involves social, emotional, cultural and economic reasons, the key is lack of knowledge and awareness of DR. Our findings have several practical implications for health policymakers and programme planners in China.

Our study had some limitations. Firstly, this study was conducted in a small sample of 27 patients, and so the potential generalisability of the findings may be limited. Secondly, 20 patients were accompanied by family members which may have caused insufficient expression of the patients' opinions. Hence further research, including a large scale quantitative study, should be conducted to better understand the problems identified in the current study.

Contributors FD was involved in question development, data collection and analysis, and manuscript preparation. YL participated in data analysis and manuscript preparation. XC was involved in question development and manuscript preparation. NC was involved in literature review and manuscript preparation. JZ was involved in question development and manuscript preparation. QC was involved in data collection and analysis. LC was involved in data collection and analysis. XC was involved in the interviews of the patients, data collection and analysis. XZ was involved in question development and manuscript preparation. CY was involved in interview of the patients, data analysis and manuscript preparation. YL was involved in question development and manuscript preparation. All authors read and approved the final manuscript.

Funding Supported by National Natural Science Foundation of China (81400381), Medical Scientific Research foundation of Guangdong Province, China (B2013140), Science and Technology Programme of Guangdong Province, China (2013B020400003) and Science and Technology Programme of Guangzhou, China (15570001).

Competing interests None declared.

Ethics approval The study was approved by the ethics committee of Zhongshan Ophthalmic Centre.

Provenance and peer review Not commissioned; externally peer reviewed.

Data sharing statement No additional data are available.

Open Access This is an Open Access article distributed in accordance with the Creative Commons Attribution Non Commercial (CC BY-NC 4.0) license, which permits others to distribute, remix, adapt, build upon this work noncommercially, and license their derivative works on different terms, provided the original work is properly cited and the use is non-commercial. See: http:// creativecommons.org/licenses/by-nc/4.0/

\section{REFERENCES}

1. $\mathrm{Xu} \mathrm{Y}$, Wang $\mathrm{L}, \mathrm{He} \mathrm{J}$, et al. Prevalence and control of diabetes in Chinese adults. JAMA 2013;310:948-59.

2. Wild S, Roglic G, Green A, et al. Global prevalence of diabetes: estimates for the year 2000 and projections for 2030. Diabetes Care 2004;27:1047-53

3. Congdon NG, Friedman DS, Lietman T. Important causes of visual impairment in the world today. JAMA 2003;290:2057-60.

4. Cheung N, Mitchell P, Wong TY. Diabetic retinopathy. Lancet 2010;376:124-36

5. Zhang X, Saaddine JB, Chou CF, et al. Prevalence of diabetic retinopathy in the United States, 2005-2008. JAMA 2010;304:649-56.

6. Wang FH, Liang YB, Zhang F, et al. Prevalence of diabetic retinopathy in rural China: the Handan Eye Study. Ophthalmology 2009;116:461-7.

7. Xie X, Xu L, Yang H, et al. Frequency of diabetic retinopathy in the adult population in China: the Beijing Eye Study 2001. Int Ophthalmol 2009;29:485-93.

8. Xu J, Wei WB, Yuan MX, et al. Prevalence and risk factors for diabetic retinopathy: the Beijing Communities Diabetes Study 6. Retina (Philadelphia, Pa) 2012;32:322-9. 
9. Klein R, Klein BE, Moss SE, et al. The Wisconsin epidemiologic study of diabetic retinopathy. II. Prevalence and risk of diabetic retinopathy when age at diagnosis is less than 30 years. Arch Ophthalmol 1984;102:520-6.

10. Klein R, Klein BE, Moss SE, et al. The Wisconsin epidemiologic study of diabetic retinopathy. III. Prevalence and risk of diabetic retinopathy when age at diagnosis is 30 or more years. Arch Ophthalmol 1984;102:527-32.

11. Wang D, Ding X, He M, et al. Use of eye care services among diabetic patients in urban and rural China. Ophthalmology 2010;117:1755-62.

12. Bragge $\mathrm{P}$, Gruen $\mathrm{RL}$, Chau M, et al. Screening for presence or absence of diabetic retinopathy: a meta-analysis. Arch Ophthalmol 2011;129:435-44.

13. Hua W, Cao S, Cui J, et al. Analysis of reasons for noncompliance with laser treatment in patients of diabetic retinopathy. Can J Ophthalmol 2013;48:88-92.

14. Paksin-Hall A, Dent ML, Dong F, et al. Factors contributing to diabetes patients not receiving annual dilated eye examinations. Ophthalmic Epidemiol 2013;20:281-7.

15. Lee DJ, Kumar N, Feuer WJ, et al. Dilated eye examination screening guideline compliance among patients with diabetes without a diabetic retinopathy diagnosis: the role of geographic access. BMJ Open Diabetes Res Care 2014;2:e000031.

16. Britten N. Qualitative interviews in medical research. BMJ 1995;311:251-3.

17. Chun KM, Chesla CA. Cultural issues in disease management for Chinese Americans with type 2 diabetes. Psychol Health 2004;19:767-85.

18. Whitmarsh I. Medical schismogenics: compliance and "culture" in Caribbean biomedicine. Anthropol Q 2009:82:447-75.

19. Lewis K, Patel D, Yorston D, et al. A qualitative study in the United Kingdom of factors influencing attendance by patients with diabetes at ophthalmic outpatient clinics. Ophthalmic Epidemiol 2007;14:375-80.

20. Yu C, Holroyd E, Cheng Y, et al. Institutional incentives for altruism: gifting blood in China. BMC Public Health 2013;13:524.

21. Thompson AC, Thompson MO, Young DL, et al. Barriers to follow-up and strategies to improve adherence to appointments for care of chronic eye diseases. Invest Ophthalmol Vis Sci 2015;56:4324-31.

22. Tong WT, Vethakkan SR, Ng CJ. Why do some people with type 2 diabetes who are using insulin have poor glycaemic control? A qualitative study. BMJ Open 2015;5:e006407.

23. DCCT/EDIC Research GroupAiello LP, Sun W, Das A, et al. Intensive diabetes therapy and ocular surgery in type 1 diabetes. N Engl J Med 2015;372:1722-33.

24. Vijan S, Hofer TP, Hayward RA. Cost-utility analysis of screening intervals for diabetic retinopathy in patients with type 2 diabetes mellitus. JAMA 2000;283:889-96.

25. Lee PP, Feldman ZW, Ostermann J, et al. Longitudinal rates of annual eye examinations of persons with diabetes and chronic eye diseases. Ophthalmology 2003;110:1952-9.

26. Robbins JM, Vaccarino V, Zhang $\mathrm{H}$, et al. Socioeconomic status and diagnosed diabetes incidence. Diabetes Res Clin Pract 2005;68:230-6.

27. Williams ED, Tapp RJ, Magliano DJ, et al. Health behaviours, socioeconomic status and diabetes incidence: The Australian Diabetes Obesity and Lifestyle Study (AusDiab). Diabetologia 2010;53:2538-45

28. Chaturvedi N, Stephenson JM, Fuller JH. The relationship between socioeconomic status and diabetes control and complications in the EURODIAB IDDM Complications Study. Diabetes Care 1996;19:423-30.

29. Antonetti DA, Klein R, Gardner TW. Diabetic retinopathy. N Engl J Med 2012;366:1227-39. 